\title{
EDITORIAL
}

For reprint orders, please contact: reprints@futuremedicine.com

\section{Traumatic brain injury and dopaminergic degeneration: the long-term risks require greater attention}

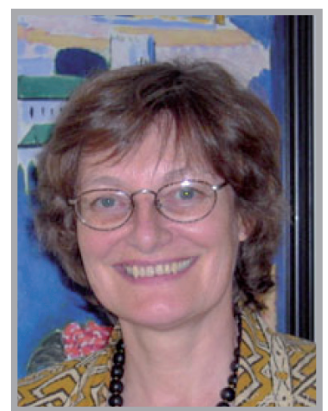

Marie-Francoise

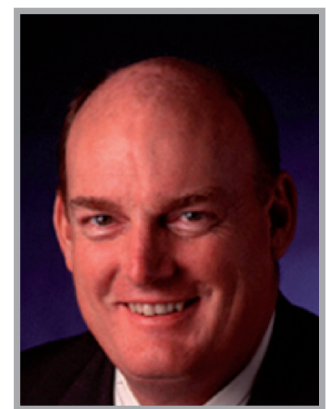

David A Hovda'

Chesselet*

Despite major strides in the treatment of the cardinal symptoms of Parkinson's disease (PD) - first with the introduction of L-Dopa in the 1960s, followed by dopaminergic agonists, modifiers of dopamine metabolism and deep-brain stimulation - the disease remains a vexing problem in neurology because of the lack of neuroprotective therapies capable of slowing or stopping disease progression [1]. Several promising agents are currently under development but so far none have been approved for clinical use [2].

One avenue actively pursued by researchers is to develop more effective neuroprotective strategies in order to better understand the pathophysiology of the disease. Over the last 10 years, genetic studies have provided important clues by identifying a series of mutations that cause familial forms of PD. Although very rare, these mutations usually point towards proteins, signaling pathways and mechanisms that are also involved in sporadic PD [3]. One example is $\alpha$-synuclein, which has been linked to PD because mutations present in a few families cause early onset PD.
Yet pathological aggregates of $\alpha$-synuclein are found in the brains of all PD patients, including in Lewy bodies, and polymorphisms in the $\alpha$-synuclein gene are highly linked to sporadic PD by genome-wide association studies [4].

However, despite these advances, the cause of sporadic PD remains unknown. Epidemiological studies have identified both environmental and genetic risk factors in causing PD, and the two very likely interact in ways that we are only beginning to unravel [5]. Such interactions contribute to the difficulty in identifying risk factors with certainty since the population examined, and the presence or absence of other conditions will influence the outcome of studies, resulting in the dismissal of some important findings as 'not reproducible'.

One such risk factor is traumatic brain injury (TBI). Several epidemiological studies have identified an increased risk to develop PD in individuals with TBI, but the association has not always been confirmed, suggesting that additional factors may modulate the association between TBI and PD [6]. Furthermore, until recently, no

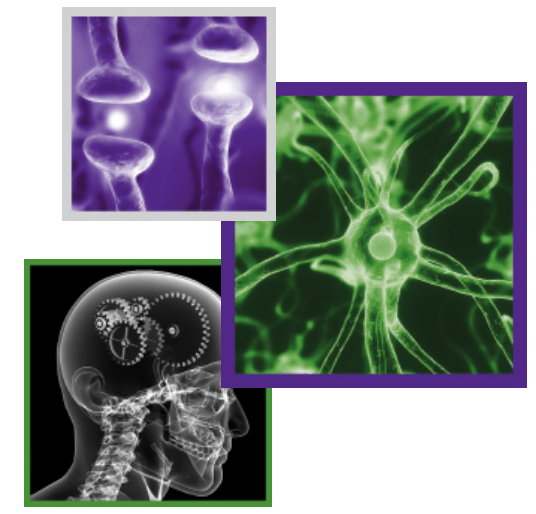

\section{“Epidemiological studies \\ have identified both environmental and genetic risk factors in causing $P D$, \\ and the two very likely interact in ways that we are only beginning to unravel.”}

'Departments of Neurology, Neurosurgery \& Molecular \& Medical Pharmacology, UCLA Brain Injury Research Center David Geffen School of Medicine, UCLA, USA

*Author for correspondence: Department of Neurology, David Geffen School of Medicine, UCLA, USA; Tel.: +1 310267 1781; mchesselet@mednet.ucla.edu 
"...a single exposure to a mild TBI, which by itself produced little evidence of loss of cells in the cerebral cortex, was sufficient to cause a progressive loss of nigrostriatal neurons..." experimental study has demonstrated an effect of TBI on nigrostriatal dopaminergic neurons, the cells that are particularly vulnerable in PD. When their loss reaches a critical threshold, the traditional symptoms of PD lead to its diagnosis.

To address this issue, our laboratories have examined the effect of a mild TBI, induced by the well-studied model of lateral fluid percussion in rats [7], on the survival of nigrostriatal dopaminergic neurons and other histological parameters observed in the brains of those with PD [8]. To our surprise, a single exposure to a mild TBI, which by itself produced little evidence of loss of cells in the cerebral cortex, was sufficient to cause a progressive loss of nigrostriatal neurons, first on the ipsilateral side and later in both hemispheres. Specifically, a 15\% loss of dopaminergic cell bodies was observed in the ipsilateral substantia nigra with rigorous stereological counting methods 11 days after the TBI, and $30 \%$ on both sides after 26 weeks. These results are interesting because they indicate that, in these experimental conditions, TBI would not have been sufficient to cause the symptoms of $\mathrm{PD}$, which require an estimated $50 \%$ loss of striatal dopaminergic neurons and $80 \%$ loss of striatal dopamine according to brain imaging studies in humans [9]. In fact, the relative preservation of the axon terminals of the nigrostriatal dopaminergic neurons we observed in the striatum at the more chronic time point suggests the presence of compensatory mechanisms. Nevertheless, a lower number of nigrostriatal dopaminergic neurons has long been considered a risk factor for PD because cell loss induced by subsequent insults may add up to reach the threshold necessary for the expression of the cardinal neurological symptoms of PD [10].

Traumatic brain injury may act as a risk factor for PD in additional ways. Indeed, we found that if the animals were exposed to an injection of paraquat, an agricultural pesticide known to increase PD risk upon chronic exposure [11], within 1 week following the TBI, then the number of nigrostriatal dopaminergic neurons lost was significantly increased. Compared with TBI alone, this combination also increased $\alpha$-synuclein and further increased inflammation, two risks factors for $\mathrm{PD}[5,12]$, in the substantia nigra. A similar synergy was not observed when the paraquat exposure was delayed 21 weeks after the TBI, suggesting a window of vulnerability. Thus, the wave of TBI-induced pathological changes that also initiates the loss of a population of dopaminergic neurons, will also make them more vulnerable to a second insult (in this case an environmental exposure). Injections of paraquat are of course quite different than exposure to the pesticide in a natural environment; however the chosen dose was insufficient to cause dopaminergic cell death by itself, yet did engage mechanisms that are also observed in PD brain, such as increased inflammation and oxidative stress [13].

These findings have important basic and clinical scientific implications supporting the need for further research. First, it will be essential to examine TBI as a risk factor for PD in conjunction with other environmental and genetic risk factors in future epidemiological studies in order to assess its full impact as a PD risk factor. Second, these data point further towards a high vulnerability period immediately following TBI, during which exposure to other disease-related insults may worsen the long-term outcome of the TBI [14]. This has critical implications for the care of TBI victims. Finally, more basic research is needed to define the mechanisms of increased vulnerability that follows TBI and to determine whether exposure to insults other than paraquat during this period of time is equally detrimental. It will be equally important to determine the window of increased vulnerability, and whether the age at which TBI occurs influences its long-term effects and the duration of increased vulnerability to another insult. This is particularly important because TBI is frequent in both young (16-25 years of age) and older individuals (over 65 years of age). The impact of different stages of brain development on the neurological consequences of TBI is still poorly understood. For younger individuals, the window of vulnerability may be essential in the success of therapies to block the full impact of the physical insult later on in life, and this may be critical for the treatment of athletes and military personnel. Conversely, older individuals may already have accumulated loss of nigrostriatal dopaminergic neurons or increased vulnerability due to chronic exposure to other risk factors during their lifetime, and this may increase their vulnerability to TBI due to a fall or other accident.

Together, epidemiological studies and this recent experimental work point to an important and perhaps causal connection between TBI and future risk of developing a devastating neurological disorder. These findings 
emphasize that more attention needs to be paid to the period of increased vulnerability following TBI, not only on the outcome of TBI itself but also on its long-term consequences, including neuronal loss at a distance from the site of impact and the risk of late life neurodegenerative disorders. This is related to the idea that TBI itself should be considered a disease [15]. Finally, they support the idea that PD is a multifactorial disease with synergy of multiple risk factors in causing PD [16] and point towards new directions to identify common mechanisms that can eventually put the dopaminergic neurons 'over the edge' and lead to neurological deficits. Identifying these mechanisms brings the hope that targeted neuroprotective strategies can be identified and that they will be beneficial in PD resulting from various causes.

\section{References}

1 Meissner WG, Frasier M, Gasser T et al. Priorities in Parkinson's disease research. Nat. Rev. Drug Discov. 10(5), 377-393 (2011).

2 Lohle M, Reichmann H. Clinical neuroprotection in Parkinson's disease - still waiting for the breakthrough. J. Neurol. Sci. 289(1-2), 104-114 (2010).

3 Bekris LM, Mata IF, Zabetian CP. The genetics of Parkinson's disease. J. Geriatr. Psychiatr. Neurol. 23(4), 228-242 (2010).

4 Shulman JM, De Jager PL, Feany MB. Parkinson's disease: genetics and pathogenesis. Annu. Rev. Pathol. 6, 193-222 (2011).

5 Gatto NM, Rhodes SL, Manthripragada AD et al. $\alpha$-synuclein gene may interact with environmental factors in increasing risk of Parkinson's disease. Neuroepidemiology 35(3), $191-195$ (2010).

6 Rugbjerg K, Ritz B, Korbo L, Martinussen N, Olsen JH. Risk of Parkinson's disease after hospital contact for head injury: population

Financial \& competing interests disclosure

$M-F$ Chesselet has received funding from the NIH, Michael J Fox Foundation, CHDI, Inc. Allon Therapeutics, Amicus Therapeutics and Isis Pharmaceuticals. Honoraria, consulting fees or travel reimbursement (last 12 months only) received from Michael J Fox Foundation, Genentech, BachmannStrauss Foundation, World Parkinson Congress, Merck, Canadian Government. Stock ownership (spouse): Adolor, Inc. Consultant (no fee received): Angiochem, Seneb, Psycho Genics and Clear Therapeutics. The authors have no other relevant affiliations or financial involvement with any organization or entity with a financial interest in or financial conflict with the subject matter or materials discussed in the manuscript apart from those disclosed.

No writing assistance was utilized in the production of this manuscript.

based case-control study. BMJ 337, A2494 (2008).

7 Thompson HJ, Lifshitz J, Marklund N et al. Lateral fluid percussion brain injury: a 15-year review and evaluation. J. Neurotrauma 22, $42-75$ (2005).

8 Hutson CB, Lazo CR, Mortazavi F, Giza CC, Hovda D, Chesselet M-F. Traumatic brain injury in adult rats causes progressive nigrostriatal dopaminergic cell loss and enhanced vulnerability to the pesticide paraquat. J. Neurotrauma 28(9), 1783-1801 (2011).

9 Stoessl AJ. Neuroimaging in Parkinson's disease. Neurotherapeutics 8(1), 72-81 (2011).

10 Langston JW. Predicting Parkinson's disease. Neurology 40 (10 Suppl. 3), 70-74 (1990).

11 Costello S, Cockburn M, Bronstein J, Zhang X, Ritz B. Parkinson's disease and residential exposure to maneb and paraquat from agricultural applications in the central valley of California. Am. J. Epidemiol. 169(8), 919-926 (2009).
12 Barnum CJ, Tansey MG. Modeling neuroinflammatory pathogenesis of Parkinson's disease. Prog. Brain Res. 184, 113-132 (2010).

13 Purisai MG, McCormack AL, Cumine S, Li J, Isla MZ, Di Monte DA. Microglial activation as a priming event leading to paraquatinduced dopaminergic cell degeneration. Neurobiol. Dis. 25(2), 392-400 (2007).

14 Jenkins LW, Moszynski K, Lyeth BG et al. Increased vulnerability of the mildly traumatized rat brain to cerebral ischemia: the use of controlled secondary ischemia as a research tool to identify common or different mechanisms contributing to mechanical and ischemic brain injury. Brain Res. 477(1-2), 211-224 (1989).

15 Masel BE, Dewitt DS. Traumatic brain injury: a disease process, not an event. J. Neurotrauma 27, 1529-1540 (2010).

16 Sulzer D. Multiple hit hypotheses for dopamine neuron loss in Parkinson's disease. Trends Neurosci. 30(5), 244-250 (2007). 